\title{
Entanglement Decay Versus Energy Change: A Model
}

\author{
Ting $\mathrm{Yu} *$ \\ Rochester Theory Center for Optical Science and Engineering, \\ and Department of Physics and Astronomy, University of Rochester, New York 14627, USA
}

(Dated: August 28, 2006)

\begin{abstract}
We present a simple quantum open system to show quantitatively how entanglement decoherence is related to the energy transfer between the system of interest and its environment. Particularly, in the case of the exact entanglement decoherence of two qubits, we find an upper bound for the energy transfer between the two-qubit system and its environments.

PACS numbers: 03.65.Yz, 03.65.Ud, 42.50.Lc
\end{abstract}

\section{INTRODUCTION}

Quantum entanglement is a central concept in the very foundation of quantum mechanics and recently has been regarded as an important resource in the emerging technologies such as quantum information processing and quantum computing [1, 2, 3]. However, real quantum systems are inevitably influenced by their surrounding environments. These unavoidable mutual interactions often result in the dissipative evolution of quantum coherence and loss of the useful entanglement. One of the prominent examples of such a disentanglement process arises in quantum registers where a set of qubits are coupled individually to their thermal environments.

It is desirable on both fundamental and practical grounds to understand entanglement decay in a timedependent sense [4, 5, , 6, 7, 8, 9, 10]. More fundamentally, decoherence processes due to the interaction with internal or external noises have been studied theoretically in many distinct cases [11, 12, 13]. However, it seems that a quantitative link between the degree of disentanglement and the amount of the energy transferred between the system of interest and its environment is still missing 14, 15].

Decoherence may arise in many different situations whenever the system of interest is not completely isolated from its environments. Typically, the examples in quantum optics include the so-called amplitude decoherence caused by spontaneous emissions of atoms and the phase decay by random disturbances of the relative phases of a quantum state. In the latter case there is no energy exchange between the system and its environment. In this Letter, our focus is on the decoherence processes caused by energy dissipations and fluctuations. We show explicitly how the energy transfer is related to a temporal disentanglement process of mixed states. Specifically, we will address a prominent question: How can energy transfer between the system and the environment be an indicator of the complete decoherence of quantum entanglement shared by two qubits. In order to analytically discuss this issue, it is only possibly to employ a sim-

${ }^{*}$ Electronic address: ting@pas.rochester.edu ple model. For our purpose, two-qubit models are ideal subjects as they embody in a compact way several mysterious elements of pure or mixed states entanglement such as Bell states and Werner states.

The Letter is organized as follows: In Sec. [II we present a two-qubit model and its solutions at finite temperature. The relation between the disentanglement process and the energy transfer is discussed in several interesting examples in Sec. IIII We conclude in Sec. IV]

\section{TWO-QUBIT MODEL}

Let us now consider a pair of qubits $A$ and $B$ that are initially in an entangled state, and we assume that the two qubits are affected by two noisy environments individually. To examine non-local relaxation we start with a generic quantum open system model. We let the two qubit systems interact with their local environments $\mathcal{E}_{A}$ and $\mathcal{E}_{B}$, which are modelled by very broadband and independent sets of harmonic oscillators. We can think of them as modes of photons or phonons. Alternatively one can say that a single environmental reservoir for qubits $A$ and $B$ is sufficiently macroscopic that its interaction with $A$ produces no effect on $B$, and vice versa. This is an important stipulation because it disallows multi-qubit decoherence-free subspaces, providing a good match with basic reality whenever spatially well-distributed qubits are utilized.

The total Hamiltonian for the systems of interest and their finite temperature environments can be formally written as follows $(\hbar=1)$ :

$$
H_{\mathrm{tot}}=H_{\mathrm{sys}}+H_{\mathrm{int}}+H_{\mathrm{env}}
$$

where

$$
\begin{aligned}
& H_{\mathrm{sys}}=\frac{1}{2} E_{A} \sigma_{z}^{A}+\frac{1}{2} E_{B} \sigma_{z}^{B} \text { and } \\
& H_{\mathrm{env}}=\sum_{\lambda} \omega_{\lambda} a_{\lambda}^{\dagger} a_{\lambda}+\sum_{\lambda} \nu_{\lambda} b_{\lambda}^{\dagger} b_{\lambda},
\end{aligned}
$$

for the Hamiltonians of the qubit system and environ- 
ments, and

$$
H_{\mathrm{int}}=\sum_{\lambda}\left(f_{\lambda}^{*} \sigma_{A} a_{\lambda}^{\dagger}+f_{\lambda} \sigma_{A}^{\dagger} a_{\lambda}\right)+\sum_{\lambda}\left(g_{\lambda}^{*} \sigma_{B} b_{\lambda}^{\dagger}+g_{\lambda} \sigma_{B}^{\dagger} b_{\lambda}\right)
$$

for the qubit-environment interactions. Here $\sigma_{A}$ and $\sigma_{B}$ are the system operators coupling each qubit to its local environment, where $f_{\lambda}$ and $g_{\lambda}$ are coupling constants and $\sigma_{z}$ denotes the usual diagonal Pauli matrix. The standard 2-qubit basis is used: $|++\rangle,|+-\rangle,|-+\rangle,|--\rangle$, where $| \pm \pm\rangle \equiv| \pm\rangle_{A} \otimes| \pm\rangle_{B}$ denote the eigenstates of the product Pauli matrix $\sigma_{z}^{A} \otimes \sigma_{z}^{B}$ with eigenvalues \pm 1 . We assume that each environment is in a thermal state with arbitrary finite temperature $T$.

Note that in the interaction Hamiltonian (4) the rotating wave approximation (RWA) is used, this ensures that the bare energy is conserved and the virtual quanta due to the presence of the counter-rotating terms do not play a role in our discussions. Besides, the approximation greatly simplifies the calculations. It should be noted that we have assumed that coupling between the atoms and photon fields is so weak such that the Markov approximation is justified.

Under usual Markov conditions the master equation for the two two-state particles (two qubits) can be derived, and moreover the solution of the master equation can be expressed in terms of 16 Kraus operators [16]:

$$
\rho(t)=\sum_{i} M_{i}(t) \rho(0) M_{i}^{\dagger}(t)
$$

where $M_{i}$ are the Kraus operators which completely describe the reduced dynamics of the qubits system interacting with the finite temperature heat baths.

We show in what follows that for a class of simple, yet important initial states it is possible to determine analytically the relation between the exact entanglement decoherence and the mean energy (or qubit inversion) of either qubit $A$ or qubit $B$. To be specific, we consider all entangled states in the very important "standard" class [17] of bipartite density matrices:

$$
\rho(t)=\left[\begin{array}{llll}
a & 0 & 0 & 0 \\
0 & b & z & 0 \\
0 & z & c & 0 \\
0 & 0 & 0 & d
\end{array}\right] .
$$

This standard class includes the well-known pure Bell states and important mixed Werner states [18, 19]. Such density matrices appear naturally, for example, in spin chain models [20, 21] and have been used in various examinations of measures of entanglement [22, 23]. Their significance is enhanced by the fact that their form is preserved under noisy decoherence evolution. That is, after an interval of interaction with a noise reservoir the only change in $\rho$ is that the non-zero elements evolve from $a, b, c, d$ and $z$, which then serve as initial values.

Under the thermal noises these initial values evolve as follows (for the zero temperature case see [8]):

$$
\begin{aligned}
a(t)= & N_{1} \gamma^{4} a+N_{2}\left[a+\omega^{2}(b+c)+\omega^{4} d\right] \\
& +N_{3}\left[2 \gamma^{2} a+\gamma^{2} \omega^{2}(b+c)\right], \\
b(t)= & N_{1}\left(\gamma^{2} b+\gamma^{2} \omega^{2} a\right)+N_{2}\left(\gamma^{2} b+\gamma^{2} \omega^{2} d\right) \\
& +N_{3}\left[b+\gamma^{4} b+\omega^{2}(a+d)+\omega^{4} c\right], \\
c(t)= & N_{1}\left(\gamma^{2} c+\gamma^{2} \omega^{2} a\right)+N_{2}\left(\gamma^{2} c+\gamma^{2} \omega^{2} d\right) \\
& +N_{3}\left[c+\omega^{2}(d+a)+\omega^{4} b+\gamma^{4} c\right], \\
d(t)= & N_{1}\left[d+\omega^{2}(b+c)+\omega^{4} a\right]+N_{2} \gamma^{4} d \\
& +N_{3}\left[2 \gamma^{2} d+\gamma^{2} \omega^{2}(b+c)\right], \\
z(t)= & \gamma^{2} z,
\end{aligned}
$$

where the numerical factors are given by $N_{1}=$ $\frac{(\bar{n}+1)^{2}}{(2 \bar{n}+1)^{2}}, N_{2}=\frac{\bar{n}^{2}}{(2 \bar{n}+1)^{2}}$ and $N_{3}=\frac{\bar{n}(\bar{n}+1)}{(2 \bar{n}+1)^{2}}$. Note that the above solution was obtained under the assumption that the two qubits are affected by the two identical local environmental noises, so we have $\gamma \equiv \gamma(t)=$ $\exp \left[-\frac{1}{2} \Gamma(2 \bar{n}+1) t\right], \omega \equiv \omega(t)=\sqrt{1-\gamma^{2}}$, where $\bar{n}$ is the mean number of quanta in the thermal reservoirs $\mathcal{E}_{A}$ and $\mathcal{E}_{B}$ and $\Gamma$ is the damping rate of the qubits $A$ and $B$.

\section{ENERGY TRANSFER AND EXACT DISENTANGLEMENT}

Despite considerable efforts made in the last decade, the efficient evaluations of entanglement are still restricted to a few classes of mixed states. In the case of two qubits, it turns out that $C(\rho(t))$, the Wootters concurrence [24], is a satisfactory (and conventional) tool for measuring the quantum entanglement, where $1 \geq C \geq 0$ gives the range between the limits of maximally entangled and fully separable, respectively. For the standard density matrix (6), the concurrence of $\rho(t)$ is given by

$$
C(\rho(t))=2 \max \{0,|z(t)|-\sqrt{a(t) d(t)}\} .
$$

We see from the above expression that the entangled state (6) may become disentangled completely $(C \rightarrow 0)$ in a finite time. In fact, this occurs if

$$
|z(t)| \leq \sqrt{a(t) d(t)} .
$$

Now it is easy to see that when $t \rightarrow \infty$, we have $a(t) \rightarrow N_{2}, d(t) \rightarrow N_{1}$, but $z(t) \rightarrow 0$. Therefore, the inequality (13) is easily satisfied for a finite $t$. By this way, we have proven that all entangled states with standard matrix form (6) will completely disentangle in finite times if exposed to finite-temperature reservoirs $(\bar{n} \neq 0)$. This is true even though, as one can easily check, the qubits $A$ and $B$ only decay exponentially to zero over an infinite time.

This simplified model allows us to determine concurrence by computing the energy change of a single qubit. For example, let us consider the energy transfer between qubit $\mathrm{A}$ and its environment. Here we define the energy 
dissipation of the qubit $A$ as: $\Delta E(t)=E(0)-E(t)$ with $E(t)=\operatorname{Tr}\left(\rho H_{A}\right)$ where $H_{A}=E_{A} \sigma_{z} / 2$. The same is true for qubit $B$. For simplicity, in this paper we will only deal with the density matrix (6), the mean energy of qubit $A$ at $t$ is then simply given by

$$
E(t)=\frac{1}{2} E_{A}\left(\alpha+\beta \omega^{2}\right),
$$

where the coefficients are $\alpha \equiv a+b-c-d$ and $\beta \equiv$ $2\left(c+d-N_{1}-N_{3}\right)$. Thus the $\omega$ function can be expressed in terms of the net local energy dissipation $\Delta E(t)$ during the time period $t$ :

$$
\omega^{2}=-\frac{\Delta E(t)}{E_{A}\left(c+d-N_{1}-N_{3}\right)} .
$$

By inserting (15) into (12), we see that entanglement evolution is entirely determined by the energy change $\Delta E$. As a consequence of (15), we will now show in the following how the energy dissipation is related to the exact entanglement decoherence.

We start with the zero-temperature case $T=0(\bar{n}=0)$ and the initial density matrix with $a=b=c=z=1 / 3$. We have shown [8] that the solution to the master equation then takes the form: $a(t)=\gamma^{4} / 3, b(t)=c(t)=$ $\left(\gamma^{2}+\gamma^{2} \omega^{2}\right) / 3, d(t)=\left(2 \omega^{2}+\omega^{4}\right) / 3$ and the disentanglement is complete at the time $\Gamma t=\ln \left(\frac{2+\sqrt{2}}{2}\right)$. Here we see that (15) becomes $\omega^{2}=3 \Delta E(t) /\left(2 E_{A}\right)$. Then from (13) we know that the energy dissipation needed for complete disentanglement is

$$
\Delta E=\frac{2}{3}(\sqrt{2}-1) E_{A} \approx 0.28 E_{A} .
$$

As another interesting case, we now consider $T \neq$ $0(\bar{n} \neq 0)$, and begin with the well-known Bell states

$$
|\Psi\rangle=\frac{1}{\sqrt{2}}(|+-\rangle \pm|-+\rangle .
$$

The above discussions have shown that the Bell states become completely disentangled in a finite time. First, note that the density matrix at $t$ for the initial Bell state takes the standard form but its time-dependent matrix elements simplify as

$$
\begin{gathered}
a(t)=N_{2} \omega^{2}+N_{3} \gamma^{2} \omega^{2}, \\
b(t)=c(t)=N_{1} \gamma^{2}+N_{2} \gamma^{2}+N_{3}\left(\gamma^{4}+1+\omega^{4}\right), \\
d(t)=N_{1} \omega^{2}+N_{3} \gamma^{2} \omega^{2}, \\
z(t)= \pm \frac{1}{2} \gamma^{2} .
\end{gathered}
$$

Next we are interested in the mean energy of qubit $A$ at $t$, which is given by

$$
\Delta E=-\frac{E_{A} \omega^{2}}{2(2 \bar{n}+1)} .
$$

Now we want to know how much energy transfer is needed in order to completely decohere the Bell states. For this purpose, let us look at the concurrence (12). Clearly, if $\gamma^{2} \leq 2 \omega^{2} \sqrt{N_{1} N_{2}}$, then (13) will be satisfied. This immediately leads to the condition,

$$
\omega^{2} \geq \frac{1}{1+2 N_{3}}=\frac{(2 \bar{n}+1)^{2}}{(2 \bar{n}+1)^{2}+2 \bar{n}(\bar{n}+1)}
$$

for the Bell states to be completely disentangled, i.e., for $C(\rho) \equiv 0$. This amounts to saying that if the energy transfer between the qubit $\mathrm{A}$ and its environment is more than $|\Delta E|_{\mathrm{ub}}$

$$
|\Delta E|_{\mathrm{ub}}=\frac{E_{A}(2 \bar{n}+1)}{2\left[(2 \bar{n}+1)^{2}+2 \bar{n}(\bar{n}+1)\right]},
$$

then the Bell states (17) are completely disentangled.

More generally for $\bar{n} \neq 0$, it can be proved that

$$
|\Delta E|_{\mathrm{ub}}^{\prime}=\frac{\left|z E_{A}\left(c+d-N_{1}-N_{2}\right)\right|}{|z|+(b+c) N_{3}}
$$

provides an upper bound for all the standard matrices (6). Namely, if the energy transfer $\Delta E$ between the system and the environment is more than $|\Delta E|_{\mathrm{ub}}^{\prime}$, then all the standard density matrices (6) become completely disentangled.

\section{CONCLUSION}

In summary, in this Letter, for a class of simple initial entangled states, we are able to analytically compute the amount of energy change that corresponds to the complete disentanglement of the two-qubit system. In the case of zero-temperature heat bath, the energy dissipation is the only source of decoherence. Our results quantitatively establish a relation between the degree of disentanglement and the energy dissipation. In the finite temperature case, we have provided an upper bound of the energy transfer that indicates the exact entanglement decoherence. The present paper only deals with the formal relation between the degree of entanglement and the energy change. The explicit exploration of the relation between energy changes and the degrees of disentanglement in the context of quantum measurement theory is certainly a very interesting issue [14, 15]. Also, it should be noted that our results are based on a master equation in the Markov regimes, it is of interest to study a more general case where non-Markovian features can be incorporated. All of these issues will be the topics of future publications. 


\section{Acknowledgments}

We gratefully acknowledge useful discussions with J.H. Eberly and Jeff Pratt. This research was initiated under a research grant for which we express appreciation to $\mathrm{L}$. J. Wang and the NEC Research Institute.
[1] M. A. Nielsen and I. L. Chuang, Quantum Computation and Quantum Information (Cambridge Univ. Press, 2000).

[2] P. Shor, SIAM J. Computing, 26, 1484(1997).

[3] L. K. Grover, Phys. Rev. Lett. 78, 325(1997).

[4] L. Diosi, in Irreversible Quantum Dynamics, edited by F. Benatti and R. Floreanini (Springer, New York, 2003), pp. 157-163.

[5] P. J. Dodd and J. J. Halliwell, Phys. Rev. A 69, 052105 (2004); P. J. Dodd, Phys. Rev. A 69, 052106 (2004).

[6] T. Yu and J. H. Eberly, Phys. Rev. B 66, 193306 (2002).

[7] T. Yu and J. H. Eberly, Phys. Rev. B 68, 165322 (2003).

[8] T. Yu and J. H. Eberly, Phys. Rev. Lett. 93, 140404 (2004).

[9] D. Tolkunov, V. Privman and P. K. Arvind, Phys. Rev. A 71, 060308(R) (2005).

[10] M. F. Santos, P. Milman, L. Davidovich, and N. Zagury, Phys. Rev. A 73, 040305(R) (2006).

[11] W. H. Zurek, Phys. Today 44, 1036 (1991); Prog. Theor. Phys. 89, 282 (1993).

[12] E. Joos and H. D. Zeh, Z. Phys. B 59, 223 (1985).
[13] B. L. Hu, J. P. Paz, and Y. Zhang, Phys. Rev. D 45, 2843 (1992).

[14] An earlier discussion on the decoherence and energy change can be found in V. Privman and D. Mozyrsky, Proc. SPIE 4047, 36-47 (2000).

[15] D. Mozyrsky, V. Privman, Mod. Phys. Lett. B 14, 303 (2000).

[16] T. Yu and J. H. Eberly, in preparation (2006).

[17] T. Yu and J. H. Eberly, e-print quant-ph/0503089

[18] R. Werner, Phys. Rev. A 40, 4277 (1989).

[19] T. Hiroshima and S. Ishizaka, Phys. Rev. A 62, 044302 (2000).

[20] J. S. Prate, Phys. Rev. Lett. 93, 237205 (2004).

[21] J. Wang, H. Batelaan, J. Podany and A. F. Starace, e-print quant-ph/0503116.

[22] W.J. Mungo, et al., Phys. Rev. A 64, 030302 (2001).

[23] N.A. Peters, T.C. Wei and P.G. Kwiat, Phys. Rev. A 70, 052309 (2004).

[24] W. K. Wootters, Phys. Rev. Lett. 80, 2245 (1998). 